\title{
Virtual Simulation Experiment Design under the Guidance of Emotional Design Theory
}

\author{
Ting Lan'1, Fuan Wen'1, Meng Huang² \\ ${ }^{1}$ School of Network Education, Beijing University of Posts and Telecommunications, Beijing, China \\ ${ }^{2}$ Beijing Ruizhirongke Holding Co. Ltd., Beijing, China \\ Email: 1553481189@qq.com
}

How to cite this paper: Lan, T., Wen, F. and Huang, M. (2019) Virtual Simulation Experiment Design under the Guidance of Emotional Design Theory. Journal of Software Engineering and Applications, 12, 51-59.

https://doi.org/10.4236/jsea.2019.123004

Received: September 14, 2018

Accepted: March 24, 2019

Published: March 27, 2019

Copyright $\odot 2019$ by author(s) and Scientific Research Publishing Inc. This work is licensed under the Creative Commons Attribution International License (CC BY 4.0).

http://creativecommons.org/licenses/by/4.0/

\begin{abstract}
In recent years, virtual simulation experiments have been widely used in education. However, at present, academic research on virtual simulation experiments mostly focuses on key technologies, and there are few emotional studies on virtual experiments. Based on the three-layer model of emotional design theory, this paper puts forward the method strategy of emotional simulation design in virtual simulation experiment, in order to provide some reference value for the design of virtual simulation experiment.
\end{abstract}

\section{Keywords}

Virtual Simulation Experiment, Emotional Design, Experimental Design

\section{Introduction}

With the development of computer technology and network technology, virtual simulation experiments have been widely used in education and teaching. However, in these virtual simulation experiments, many experiments lack emotional design and cannot mobilize students' enthusiasm. In academia, papers on virtual simulation experiments are also emerging. In foreign countries, Amirkhani designed and developed a virtual laboratory with audiovisual and force feedback, and experimentally verified the effectiveness of virtual experiments. Shangfu, Hao proposed a virtual experiment design method based on object-oriented thinking, which provides students with free experimental practice. In China, $\mathrm{Xu}$ Yifan expounded the conception, design and implementation process of middle school physics simulation experiments, which effectively promoted the development and popularization of virtual experiments. Xu Juanjuan combined with the current case of physics experiment teaching, under the guidance of relevant theories, put forward the relevant strategies of physical virtual simulation expe- 
riment design, which made up for the shortcomings of current physical virtual simulation experiment design.

This paper analyzes the design process of virtual simulation experiment, and combines the three levels of emotional design theory to propose a strategic plan for emotional simulation design of virtual simulation experiment. Through the method of questionnaire survey, the reliability and effectiveness of the strategy are verified, in order to provide valuable reference for the designers of virtual simulation experiments, so that the design of virtual simulation experiments can be more standardized and emotional. The first part of the article describes the background and current situation of the virtual simulation experiment. The second part gives a brief summary of the virtual simulation experiment. The third part elaborates the related concepts of emotional design. The fourth part proposes the related concept of emotional design. The design strategy of the virtual simulation experiment, the fourth part is the core part of the paper, and the last part summarizes the article.

\section{Virtual Simulation Experiment Overview}

\subsection{Introduction to Virtual Simulation Experiments}

The virtual simulation experiment is based on virtual reality technology and modern information technology. Based on the hierarchical and modular design ideas, the software and hardware are combined to create a virtual environment similar to the real world, supplemented by network technology. Experimental mode is on a personal computer or on a virtual device [1]. The virtual simulation experiment studied in this paper runs on the PC side, and is mainly used for students' pre-school preparation or self-learning under the class.

\subsection{Design Process of Virtual Simulation Experiment}

The design process of the virtual simulation experiment should be carried out in accordance with the instructional design, virtual scene design, virtual object design, interaction design and evaluation design.

Instructional design: Designing a virtual simulation experiment is like designing a course. First, teaching design should be carried out. Good teaching design can stimulate students' curiosity and improve learning performance.

Virtual scene design: The experimental scene is where the students do the experiment, which determines the basic tone and layout of the whole experiment. The experimental scene is the first impression of the learner on the experiment. Therefore, the design of the experimental scene is very important.

Virtual object design: The virtual object refers to the various instruments and equipment used in the experiment, and is the main interaction object of the learner. After the experimental object is designed, the construction of the whole experimental environment is basically completed.

Interaction design: Including the design of the learner's experimental process, the design of the mouse pointer shape, the interaction design of the interface na- 
vigation, the good interaction mode can mobilize the students' curiosity and curiosity, and play a multiplier role.

Evaluation design: Evaluation design refers to the absolute evaluation given by the students after the completion of the experiment and the self-evaluation of the students. The evaluation can encourage the learners to think and enable the learners to internalize the knowledge more quickly.

After completing the above five steps, the design of the virtual simulation experiment has been basically completed. The design process is shown in Figure 1.

\section{Emotional Design Overview}

\subsection{The Concept and Connotation of Emotional Design}

The concept of emotional design was proposed by Donald A. Norman in "Emotional Design", which originated in the field of cognitive psychology and was originally used in product design to reveal the relationship between human emotions and products. Subtle relationship [2]. The so-called emotional design is to consider the changes of people's emotions when designing products. When designing products, grasp the details of each product, so that users can get emotional satisfaction while experiencing products.

As an educational product, virtual simulation experiment also follows the concept of emotional design. When designing virtual simulation experiment, we should consider the emotional factors of learners, explore the emotional needs of learners from the perspective of learners, and design emotional simulations. The concept is applied to the design of virtual simulation experiments.

\subsection{Emotional Design Hierarchical Model of Virtual Simulation Experiment}

From the perspective of psychology, Norman proposed a hierarchical model of emotional design, namely the instinct layer, the behavior layer, and the reflection layer [3]. The instinct layer refers to the stimulation of the product's senses, including hearing, vision, touch, taste, etc. The instinct layer can bring the user the initial and most intuitive feeling, and is the user's first impression of the product. The behavior layer refers to the realization process of the product function, the user's operation on the product, and the product feedback to the user belong to the scope of the behavior layer. The behavior layer can enable the user to quickly understand the usage mode of the product and help the user to smoothly use the product, thereby enabling the user to obtain for a better experience, the ease of use and usability of the product are reflected at this level. Reflective layer refers to the user's association, summary, reasoning, reflection, etc. A good product must be able to touch the user to think, including emotional activities such as recall, reflection, association, etc. [4].

Figure 1. Design process of virtual simulation experiment. 
Combined with the hierarchical model of emotional design, the design process of virtual simulation experiments can be summarized. Virtual scenes and virtual objects are presented to learners in a visual and auditory manner, giving learners a first impression, and therefore belong to the design of the instinct layer. The interaction design refers to the learner's operation of the experimental instrument and the interaction design with the interface navigation, which belongs to the design of the behavior layer. Evaluation design is used to inspire learners to actively reflect and summarize, belonging to the highest level of emotional design, so evaluation design is a reflection layer. The corresponding relationship between the design process of the virtual simulation experiment and the threelayer model of emotional design is shown in Figure 2.

\subsection{The Significance of Emotional Design in Virtual Simulation Experiment}

The emotional design of virtual simulation experiments can bring a happy, happy and confident emotional experience to learners. This kind of emotion can motivate students' enthusiasm, especially for students with weak foundation and no interest in learning. In the virtual simulation experiment system, the influence of emotions comes from intangibles, which can immerse learners in the unconsciously, thus completing the experiment step by step, gaining a sense of joy and achievement, and making up for the boring status of traditional teaching. The emotional design of virtual simulation experiments will also be beneficial to its promotion and application in the field of education, and is the development trend of virtual simulation experiments.

\section{Emotional Design Strategy of Virtual Simulation Experiment}

\subsection{Teaching Design Emotional}

Teaching design is the first step of virtual simulation experiment design, including the design of teaching objectives, key and difficult points analysis, teaching content and teaching process. The teaching design should follow the idea of "people first", consider the needs of learners from the perspective of learners,

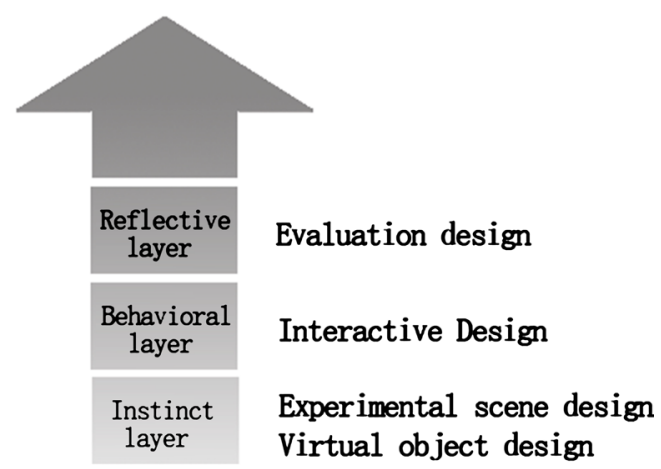

Figure 2. Correspondence between the design process of virtual simulation experiment and the three-layer model of emotional design. 
and in the arrangement of teaching process, guide learners to conduct independent exploration as far as possible, from the superficial to the deep, and continuously go deep, so that learners can acquire knowledge and skills unconsciously [4]. Before the experiment, learners can understand the target and key difficulties of the experiment, which can help learners to learn in a targeted way. At the end of the experiment, students should be guided to fill in the experiment report, which can help learners to sort out and summarize the knowledge, and then feel the sense of achievement and satisfaction of obtaining knowledge.

\subsection{Instinct Layer Design Strategy}

1) Match colors reasonably

Color influences the user's first impression and is the most intuitive and instinctive experience for the user. When matching colors, try to be scientific and reasonable. In addition to considering the aesthetics of the interface, you should also consider the characteristics of the virtual simulation experiment and the characteristics of the learner [5]. For example, the experimental types are different, the color of the experimental interface is different, the biological experiment is more green, the physical and chemical experiments are more blue, the primary school students prefer the colorful, cartoon-like interface, while the middle school students prefer the calm color.

2) Set up a "real" experiment

The so-called virtual simulation experiment is to provide the learner with an experience similar to the real experimental environment, so that the seamless connection between the real world and the virtual world can be realized, and the learner does not have a strange feeling in the virtual scene, in the virtual simulation experiment. The acquired methods and skills can also be moved to real experiments more quickly. Therefore, the construction of the experimental scene is as far as possible in the real world scene.

3) Add sound effects as appropriate

Sound effects can often play a role in setting the atmosphere. In virtual simulation experiments, sound effects can be added appropriately, such as the sound of instrument collisions and the sound of water flow. For some boring experiments, you can also add background music [6]. Background music enables learners to blend into the scene more quickly, creating a feeling of comfort and pleasure, which can better guide learners to experiment.

\subsection{Behavior Layer Design Strategy}

1) Improve the interactive experience

In the virtual simulation experiment environment, the learner needs to interact with the experimental object. The virtual simulation experiment studied in this paper runs on the PC side, and the experimental object is operated by mouse and keyboard. Compared with the real experimental process, the virtual simulation experiment based on PC terminal has Many limitations. Therefore, when 
designing the interaction mode of virtual objects, the virtual objects should be able to implement basic translation and flipping under the manipulation of more natural input devices [7]. Learners are free to adjust the perspective of the experimental environment and roam. Only when the interaction method is improved can the learner be put into the virtual simulation experiment and the experiment can be completed smoothly.

2) Add intelligent guidance function

The behavioral layer is designed to encourage learners to complete experiments and gain a sense of accomplishment and satisfaction. Students encounter problems during the experiment, and should not and cannot tell the students the correct answers, and the intelligent guidance system can provide timely, accurate and appropriate prompts. Intelligent guidance is conducive to students' selfdirected learning. It can guide students to think constantly during the experiment and cultivate students' independent consciousness, thus bringing students the same emotional experience as the teachers around them. Only when the students successfully complete the experiment can they carry out the induction and summary of the reflective layer.

\subsection{Reflective Layer Design Strategy}

1) Feedback experiment results

The reflective layer is the highest level of emotional design, emphasizing the learner's understanding, induction and reasoning of knowledge. In order to ensure the smooth progress of this process, after the experiment is over, the experimental results can be fed back to the students. The experimental results are scored and commented. The two-part composition can make the learner understand the current knowledge, and cause the learner to reflect. The comment section gives the students the next step of learning, which helps students to develop learning plans and think at a higher level.

2) Post-experimental testing

After the students complete the experiment, they can set up exercises through the exercises to consolidate the knowledge points. Exercises can motivate students to review knowledge and help them develop good habits to summarize and think. When setting up exercises, you should put the difficulty of the exercises, difficult exercises will make students lose confidence, produce bad emotions for learning, and too simple questions cannot help students find weak points of knowledge, and even make students produce Conceited emotions. Therefore, the design of the exercises should be difficult and moderate, suitable for most students to learn.

\section{Empirical Research}

\subsection{Research Design}

This paper selects 40 students of the electronic science and technology of the School of Electronic Engineering of Beijing University of Posts and Telecommu- 
nications as the research object. They have the foundation of virtual simulation experiment and have a certain understanding of the virtual simulation experiment. Through questionnaires, learn about students' opinions and opinions on rational matching colors, setting up "real" experimental scenes, properly adding sound effects, enhancing interactive experience, increasing intelligent guiding functions, feedback experimental results, and post-experiment testing design strategies. The reliability and validity of the collected questionnaires were analyzed to ensure the reliability and validity of the questionnaire data.

\subsection{Survey Design}

The questionnaire uses the Likert "five-point" scale. According to the degree of support of the learner to a certain strategy, it is divided into five options: very agree, agree, general, disagree, and disagree. The scores are 5, 4, 3, 2, and 1, respectively. The higher the score, the higher the degree of recognition of the strategy by the students. The questionnaire is divided into two parts. The first part is the basic situation of students using virtual simulation experiments. There are three questions. The second part has seven questions, each of which corresponds to a design strategy. Finally, the effectiveness of the corresponding strategy is quantified by analyzing the scores of each strategy in the questionnaire.

\subsection{Trust Level Analysis}

Reliability refers to reliability, and reliability analysis is an important way to measure the reliability and stability of a questionnaire. This study conducted a reliability analysis of the seven strategies of virtual simulation experiment design, and analyzed the Krumbach coefficient of the questionnaire in SPSS. Determine the reliability of the questionnaire based on the range of the Krumbach coefficient. As shown in Figure 3, the results of the representative questionnaire are very reliable.

\subsection{Validity Analysis}

Validity means that the method or means of measurement can accurately reflect the degree of the measured things. This study analyzed the value of the questionnaire in SPSS. The closer KMO is to 1, the more suitable for factor analysis. As shown in Figure 4, the value of KMO is 0.808 , which means that the questionnaire can reflect the measured things more accurately.

\subsection{Analysis of Survey Results}

A total of 40 questionnaires were distributed in this study, 30 were collected, and

Reliability Statistics

\begin{tabular}{|r|r|}
\hline $\begin{array}{c}\text { Cronbach's } \\
\text { Alpha }\end{array}$ & N of ltems \\
\hline .877 & 7 \\
\hline
\end{tabular}

Figure 3. Krumbach coefficient analysis. 
KMO and Bartlett's Test

\begin{tabular}{|ll|r|}
\hline Kaiser-Meyer-Olkin Measure of Sampling Adequacy. & .808 \\
Bartlett's Test of & Approx. Chi-Square & 86.907 \\
Sphericity & df & 21 \\
& Sig. & .000 \\
\hline
\end{tabular}

Figure 4. KMO value analysis.

Table 1. Virtual simulation experiment design strategy score.

\begin{tabular}{cc}
\hline Design Strategy & Score \\
\hline Reasonable color matching & 3.40 \\
Build a "real" experimental scene & 3.46 \\
Add sound effects as appropriate & 3.86 \\
Improve the interactive experience & 3.93 \\
Increase the intelligent guidance function & 4.15 \\
Feedback experiment results & 4.10 \\
Post-experiment test & 3.73
\end{tabular}

the invalid questionnaire was 0 . Through the analysis of the questionnaire, it was found that the scores of "adding intelligent guidance function" and "feedback experimental results" were higher. Secondly, "appropriately add sound effects", "improve interactive experience", "post-test test", and finally "reasonable matching color" and "build a real experimental scene", the scores are shown in Table 1. Therefore, when designing virtual simulation experiments, we should pay attention to intelligent guidance and feedback experimental results, and should also add sound effects, enhance interactive experience and increase post-experimental testing. These strategies can encourage students to actively complete experiments and conduct experimental reflections. Finally, pay attention to the details of the experimental design, such as the deployment of the interface color, the construction of the experimental environment, which can affect the mentality of the students in the invisible.

\section{Summary and Prospect}

Through the analysis of the questionnaire, we can summarize the results as: enhance the interactivity, create a "real" experimental environment, increase the sound effect, and give experimental feedback. Post-experimental testing is an effective virtual experimental design strategy. This experimental design strategy can motivate students and improve their academic performance. Through the research in this paper, the emotional design of the virtual simulation experiment has been preliminarily elaborated. In the future research, more attention should be paid to the deeper details of the virtual simulation experiment. Constantly, excavating new strategies and methods makes virtual simulation experiments more and more emotional. 


\section{Conflicts of Interest}

The authors declare no conflicts of interest regarding the publication of this paper.

\section{References}

[1] Xu, Y.F. (2014) Design and Implementation of Physics Simulation Experiment in Middle School Based on Virtual Reality. Hunan University, Changsha.

[2] Zheng, W.D. (2014) Emotional Design of Micro-Course: Concept, Connotation, Model and Strategy. China Audiovisual Education, 6, 101-106.

[3] Li, T.T. and Ma, X.F. (2010) Exploration of Emotional Design of Educational Games. Modern Educational Technology, 20, 32-34.

[4] Huang, M. and Wen, F. (2016) Research on the Emotional Design of Panoramic Virtual Campus. Software, 37, 1-3.

[5] Amirkhani, S. and Nahvi, A. (2016) Design and Implementation of an Interactive Virtual Control Laboratory Using Haptic Interface for Undergraduate Engineering Students. Computer Applications in Engineering Education, 24.

[6] Li, J.J. (2012) Design and Research of Physical Virtual Simulation Experiment System. Yangzhou University, Yangzhou.

[7] Hao, S.F. and Sun, B.L. (2010) The Virtual Experiment Design of Arithmetic Unit Based on Object-Oriented Technology. 2010 International Conference on MultiMedia and Information Technology, MMIT, 159-161.

https://doi.org/10.1109/MMIT.2010.189 